\title{
How to Improve the Use of Controlled Experiments as a Means for Early Technology Transfer
}

\author{
Andreas Jedlitschka
}

As stated by Robert Glass in Communications of the ACM [1], there seems to exist a gap between the information needed by industrial people and the information provided by researchers. We have, on the one hand, investigated the information need of industrial decision makers and, on the other hand, surveyed a set of controlled experiments with regard to their entropy (defined as a measure for relevant information content). The studies among industrial decision makers confirm the need for information regarding SE technology's impact on overall project cost, quality and schedule, whereas the survey of controlled experiments indicates that this information is hardly available. Accepting the importance of controlled experiments for our discipline, we conclude that in order to improve their wide-spread acceptance (usage and usability) by industrial decision makers, it is important to provide at least minimum information with regard to their need.

In terms of currently available reports of controlled experiments, we observed that the aforementioned problem is not the only one. A further problem has been identified, which hinders the widespread acceptance of the study results: It is difficult to locate relevant information. Therefore, we would like to enforce the use of reporting guidelines, which are expected to support a systematic, standardized presentation of empirical research, thus improving reporting in order to support readers in (1) finding the information they are looking for, (2) understanding how an experiment is conducted, and (3) assessing the validity of its results. We argue [2] that especially for the first issue, currently available guidelines do not provide the level of support that would be necessary for improving the use of controlled experiments as a means for early technology transfer. Whereas publishing reports of controlled experiments for the research community seems to be straightforward, the situation is different for publishing results for practitioners. They certainly care about the context, the overall results, and transfer issues, but do they care about the design and statistical analysis? Therefore, we foresee that there will be no one-size-fits-all guideline, but rather different guidelines for different user groups and purposes, e.g., for researchers aiming at aggregation or for practitioners aiming at decision support.

\section{References}

[1] Glass, R.L.: Matching Methodology to Problem Domain; In Column Practical Programmer in Communications of the ACM /Vol. 47, No. 5, May 2004, pp. 19-21

[2] Jedlitschka, A.; Pfahl, D.; Reporting Guidelines for Controlled Experiments in Software Engineering; ; In Proc. of ACM/IEEE Intern. Symposium on Software Engineering 2005 (ISESE2005), Noosa Heads, Australia, Nov 2005, IEEE CS, 2005, pp. 95-104 